\title{
Male-female differences in the number of reported incident dengue fever cases in six Asian countries
}

Martha Anker and Yuzo Arima ${ }^{b}$ on behalf of Emerging Diseases Surveillance and Reponse, Division of Health Security amd Emergencies, World Health Organization Regional Office for the Western Pacific

Correspondence to Yuzo Arima (e-mail: arimay@wpro.who.int)

Introduction. Demographic factors, such as age and sex, are associated with the likelihood of exposure to Aedes aegypti, the vector for dengue. However, dengue data disaggregated by both sex and age are not routinely reported or analyzed by national surveillance systems. This study analysed the reported number of incident dengue cases by age and sex for six countries in Asia.

Methods. Data for the Lao People's Democratic Republic, the Philippines, Singapore and Sri Lanka were obtained from DengueNet; the number of male and female dengue cases was available for four age groups ( $<1,1-4,5-14$ and $\geq 15$ years) over a cumulative period of six to 10 years. Data for Cambodia (2010) and Malaysia (1997-2008) were obtained from their respective ministries of health.

Results. An excess of males was found among reported dengue cases $\geq 15$ years of age. This pattern was observed consistently over several years across six culturally and economically diverse countries.

Discussion. These data indicated the importance of reporting data stratified by both sex and age since collapsing the data over all ages would have masked some of the male-female differences. In order to target preventive measures appropriately, assessment of gender by age is important for dengue because biological or gender-related factors can change over the human lifespan and gender-related factors may differ across countries.

$\mathrm{D}$ engue is a tropical and subtropical mosquitoborne infection that can cause severe illness and death. During the last 30 years, dengue fever has dramatically expanded its geographical range and shortened its epidemic cycle in many places. According to the World Health Organization (WHO), dengue is endemic in over 100 countries and approximately twofifths of the world's population is currently at risk for dengue fever with an estimated 50 million infections annually. ${ }^{1}$ Among the estimated 2.5 billion people at risk globally for dengue, about 1.8 billion (i.e., more than $70 \%$ ), reside in Asia Pacific countries. ${ }^{2}$

The most important vector for dengue, Aedes aegypti, is a predominantly urban mosquito species that favours particular environments such as locations where water storage is common and waste disposal services are inadequate. ${ }^{1,3}$ While exposure to such environments may be related to specific demographic factors such as age and sex, there is a scarcity of sex-specific dengue data. Indeed, sex-disaggregated dengue data are not routinely reported or analysed by surveillance systems. The few studies from Asia, such as those from Singapore, that have examined male and female dengue incidence, have tended to find greater male incidence. ${ }^{4}$ Differences in dengue incidence have been attributed to genderrelated differences in exposures such as time away from home. ${ }^{4,5}$ As gender roles, and thus exposures, change over the human lifespan, it is important to examine dengue cases by both sex and age. While there are recent studies that provide age- ${ }^{6}$ or sex-specific ${ }^{3}$ incident dengue surveillance data, few studies provide incident dengue data stratified by both age and sex. This study describes the reported number of incident dengue cases by age and sex for six countries in Asia.

\section{METHODS}

The current study analysed national surveillance data on the annual number of reported dengue cases by age and sex for six Asian countries. Reported cases included all

\footnotetext{
School of Public Health and Health Sciences, University of Massachusetts, Amherst, Massachusetts, United States of America

${ }^{b}$ World Health Organization Regional Office for the Western Pacific, Manila, the Philippines

Submission date: 24 February 2011 ; Publication date: 30 June 2011

doi: 10.5365/wpsar.2011.2.1.002
} 
reported dengue fever cases, dengue haemorrhagic fever cases and cases of dengue shock syndrome. The six countries were selected because their data either were available in DengueNet (part of the WHO Global Atlas that contains national data on select health conditions provided by the ministries of health of participating countries $^{7}$ ) or were provided to the WHO Western Pacific Regional Office by their ministries of health. Data for the Lao People's Democratic Republic, the Philippines, Singapore and Sri Lanka were from DengueNet. For these four countries, the number of male and female dengue cases was reported for four age groups $(<1,1-4,5-14$, and $\geq 15$ years) over a period of six to 10 years. Data from Cambodia and Malaysia were provided directly to WHO by their ministries of health. For Cambodia, male and female cases for age groups $\leq 4,5-9$, $10-14$, and $\geq 15$ years were available for 2010 (through 24 December 2010). For Malaysia, the reported number of dengue cases from 1997 to 2008 was available by both sex and age-group separately but not in a form stratified for both. Subnational data were available for four countries. This included the 18 provinces of the Lao People's Democratic Republic, the 15 states of Malaysia (for 2007 and 2008), the 16 regions of the Philippines and the nine provinces of Sri Lanka. For the Philippines, DengueNet used the same administrative regions that were in existence in 1998, the first year data were submitted to DengueNet from the Philippines.

Since the implications of sex and gender for infectious disease differ with age, age groups were examined separately. Sex refers to biological characteristics of males and females while gender refers to male and female norms that are socially and culturally constructed. For each country and age group, $\chi^{2}$ goodness of fit tests were used to compare the observed proportion of males among reported dengue cases to the expected proportion of males among reported cases based on the age group specific sex distribution of the national population. Demographic data for the year 2000 from the United Nations ${ }^{8}$ were used to calculate the proportion of males in the national population for each age group for which we had reported dengue data (namely $<1,1-4,5-14$ and $\geq 15$ years for the four DengueNet countries, and 0-4, 5-14 and $\geq 15$ years for Cambodia, and all ages combined for Malaysia). The United Nations report included the numbers of males and females in five-year age groups. For the $5-14$ and the $\geq 15$ year age groups, the numbers of males and females in their respective age groups were summed. For age $<1$ year, numbers of males and females were calculated as the mean of the numbers of males and females born and the numbers of males and females expected to be alive at the end of one year using data on the sex ratio at birth, number of births and sex-specific infant mortality rates from the United Nations. ${ }^{8}$ The numbers of males and females in age group 1-4 years were then calculated by subtracting those $<1$ year from those aged 0-4 years.

The consistency over time and location of differences between the proportion of males among reported cases and the proportion of males in the general population was examined in two ways. First, variations in annual data were examined and data for each year were tested for statistical significance. Second, for the $\geq 15$ year old age group in which the percent male among reported cases was found to be significantly higher than the percent male in the general population in all countries, subnational data were examined to see how consistently the excess of males was reported in subnational areas. No statistical tests were conducted on subnational data as age- and sex-stratified population figures were not available for subnational administrative regions.

\section{RESULTS}

The total number of reported dengue cases and the proportion of reported cases that were male by age group for the Lao People's Democratic Republic, the Philippines, Singapore, Sri Lanka and Cambodia were calculated (Table 1). The proportion of men in the general population for each age group is also provided for comparison. The number and percent male of dengue cases for each subnational administrative region of the Lao People's Democratic Republic, the Philippines and Sri Lanka, for the $\geq 15$ year age group and for all age groups combined were calculated (Table 2). Results for Malaysia are presented separately (Table 3 ).

\section{The Lao People's Democratic Republic}

Male-female ratios in dengue cases reported from the Lao People's Democratic Republic were variable over time and location (Tables 1 and 2). From 2000 to 2006, among infants, percent male of reported cases was similar to the percent male in the general population, and no significant differences were found for any year or for all years combined. There was a small excess of reported male cases among 1-4 year olds, and this male excess was statistically significant for all years combined 
Table 1. Reported number of dengue cases, percent male of reported cases, and percent male in the general population, by age group and year for the Lao People's Democratic Republic, the Philippines, Singapore, Sri Lanka and Cambodia ${ }^{a}$

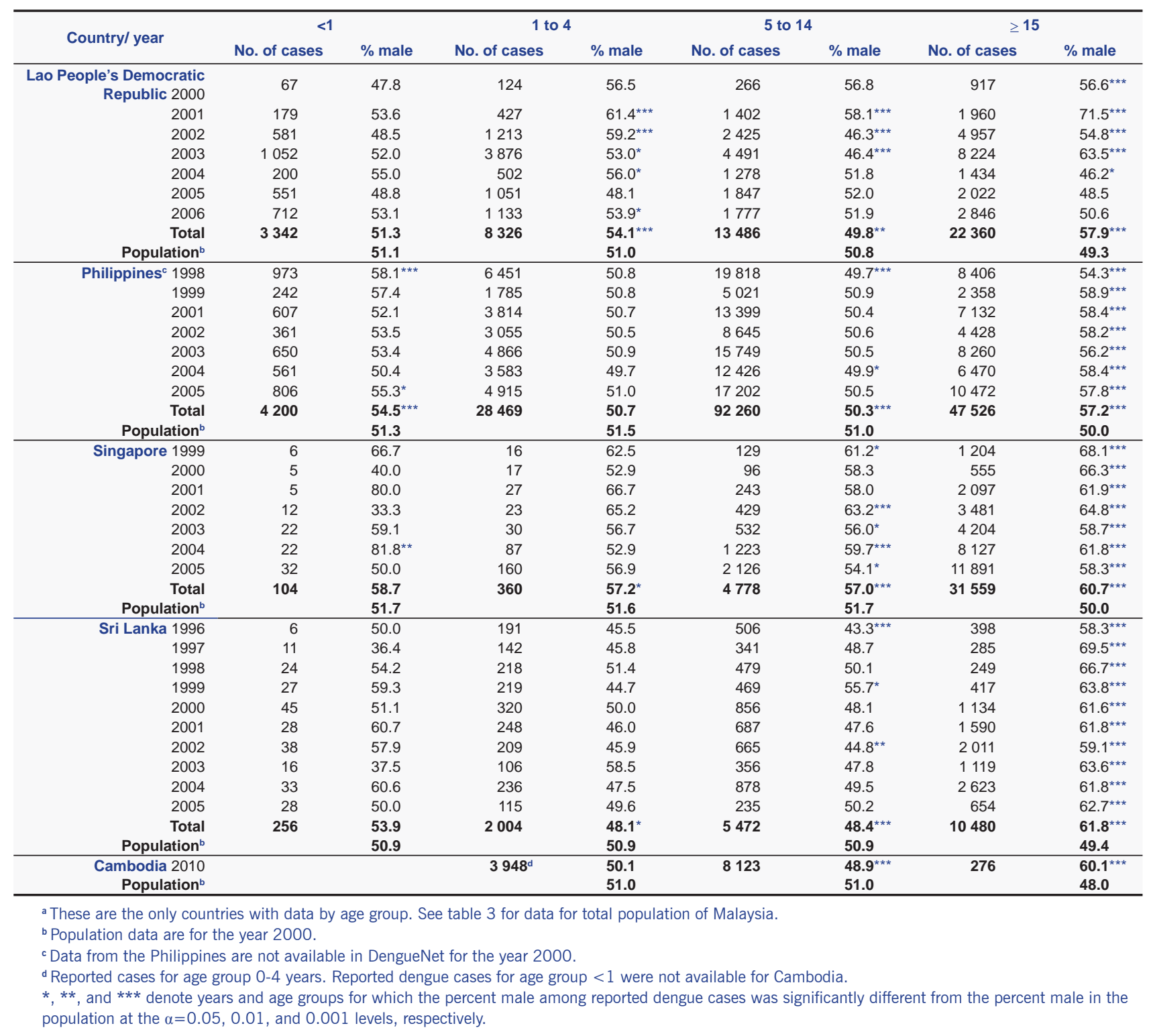

and for five of the seven years (Table 1). For 5-14 year olds, there was a slightly lower than expected proportion of males among reported cases. However, results were inconsistent from year to year, with some years reporting proportionately more and others proportionately fewer male cases than expected. The most pronounced difference in the Lao People's Democratic Republic was found among those $\geq 15$ years of age $(58 \%$ male cases compared to $49 \%$ male in the general population); a significant male excess in this age group was observed in four of the seven years assessed. Much of the male excess in this age group was driven by the larger than expected male cases who were $\geq 15$ years of age in the regions of Savannakhet Province and Vientiane Municipality (Table 2).

\section{The Philippines}

The age and sex distribution of reported dengue cases was consistent for 1998-1999 and 2001-2005 in the Philippines. Reported number of cases by age and sex was not available for the year 2000. Over these seven years, there was a significant excess of male cases among those $\geq 15$ years of age and among infants (Table 1 ). Subnational data on the sex distribution of cases $\geq 15$ years of age were also consistent, showing an 
Table 2. Number and percent male of reported dengue cases in the $\geq 15$ year age group and for all ages combined for the regions of the Lao People's Democratic Republic, 2001-2005, the Philippines, 1998-1999 and 2001-2005 and Sri Lanka, 1996-2003

\begin{tabular}{|c|c|c|c|c|c|}
\hline Country & Administrative region or province & $\begin{array}{l}\text { Number of cases, } \\
\text { age } \geq 15 \text { years }\end{array}$ & $\begin{array}{l}\% \text { male cases, } \\
\text { age } \geq 15 \text { years }\end{array}$ & $\begin{array}{c}\text { Number of cases } \\
\text { all ages }\end{array}$ & $\begin{array}{c}\% \text { male cases, } \\
\text { all ages }\end{array}$ \\
\hline \multirow{20}{*}{$\begin{array}{l}\text { Lao People's } \\
\text { Democratic } \\
\text { Republic }^{b}\end{array}$} & Attapeu & 95 & 42.1 & 269 & 46.1 \\
\hline & Bokeo & 18 & 44.4 & 68 & 57.4 \\
\hline & Borikhamxay & 1140 & 49.7 & 2837 & 50.9 \\
\hline & Champasack & 2557 & 44.0 & 6358 & 48.0 \\
\hline & Huaphanh & 14 & 71.4 & 25 & 60.0 \\
\hline & Khammuane & 1310 & 61.0 & 3132 & 52.9 \\
\hline & Luangnamtha & 0 & 0.0 & 9 & 55.6 \\
\hline & Luangprabang & 558 & 40.7 & 1777 & 49.0 \\
\hline & Oudomxay & 5 & 40.0 & 12 & 33.3 \\
\hline & Phongsaly & 0 & 0.0 & 2 & 50.0 \\
\hline & Saravane & 83 & 54.2 & 279 & 50.9 \\
\hline & Savannakhet & 7894 & 64.3 & 14080 & 57.8 \\
\hline & Sekong & 26 & 38.5 & 69 & 52.2 \\
\hline & Vientiane & 1016 & 52.3 & 2705 & 54.2 \\
\hline & Vientiane Municipality & 7319 & 59.4 & 14719 & 56.3 \\
\hline & Xayabury & 287 & 46.0 & 1082 & 51.3 \\
\hline & Xaysomboun Special Rega & 22 & 59.1 & 57 & 56.1 \\
\hline & Xiengkhuang & 3 & 66.7 & 3 & 66.7 \\
\hline & All regions combined & 22347 & 57.9 & 47483 & 54.5 \\
\hline & $\%$ Male in population $\geq 15$ years & & 49.3 & & \\
\hline \multirow{18}{*}{ Philippines $^{b}$} & Autonomous Region of Muslim Mindanao & 333 & 58.3 & 1106 & 53.4 \\
\hline & Bicol & 2687 & 52.9 & 8713 & 51.7 \\
\hline & Cordillera Administrative Region & 2238 & 55.7 & 3668 & 55.0 \\
\hline & Cagayan Valley & 3292 & 54.6 & 10049 & 52.1 \\
\hline & Caraga & 1062 & 59.0 & 3717 & 52.5 \\
\hline & Central Luzon & 8073 & 59.9 & 25274 & 53.5 \\
\hline & Central Mindanao & 2442 & 57.7 & 5975 & 53.1 \\
\hline & Central Visayas & 3552 & 57.4 & 20120 & 50.9 \\
\hline & Eastern Visayas & 2463 & 55.5 & 11399 & 52.0 \\
\hline & llocos & 632 & 56.1 & 3847 & 52.7 \\
\hline & National Capital Region & 8673 & 58.6 & 27946 & 53.7 \\
\hline & Northern Mindanao & 3193 & 55.4 & 14811 & 51.0 \\
\hline & Southern Mindanao & 3174 & 56.7 & 12256 & 51.0 \\
\hline & Southern Tagalog & 1498 & 63.5 & 7541 & 52.9 \\
\hline & Western Mindanao & 2593 & 52.7 & 7360 & 51.3 \\
\hline & Western Visayas & 1545 & 54.7 & 8506 & 51.7 \\
\hline & All regions combined & 47450 & 57.1 & 172288 & 52.3 \\
\hline & $\%$ Male in population $\geq 15$ years & & 50.0 & & \\
\hline \multirow{11}{*}{ Sri Lanka ${ }^{\mathrm{bc}}$} & Central & 666 & 63.5 & 1163 & 56.7 \\
\hline & Eastern & 209 & 61.7 & 428 & 62.4 \\
\hline & North Central & 236 & 68.2 & 346 & 63.0 \\
\hline & North Western & 730 & 64.0 & 1117 & 63.3 \\
\hline & Northern & 149 & 59.7 & 187 & 59.4 \\
\hline & Sabaragamuwa & 434 & 63.4 & 645 & 58.1 \\
\hline & Southern & 953 & 65.9 & 1440 & 59.0 \\
\hline & Uva & 132 & 66.7 & 213 & 57.3 \\
\hline & Western & 3349 & 59.1 & 7407 & 53.9 \\
\hline & All regions combined & 6858 & 61.8 & 12946 & 55.9 \\
\hline & $\%$ Male in population $\geq 15$ years & & 49.4 & & \\
\hline
\end{tabular}

a Reported numbers of dengue cases by sex missing for Xaysomboun Special Region for the year 2006.

${ }^{\mathrm{b}}$ Total number of cases reported from regions was less than the total number reported at the national level in Table 1.

c No provincial data available for Sri Lanka for the years 2004 and 2005.

excess of male cases in all regions in this age group (Table 2). For the 1-4 and 5-14 year age groups, the proportions of males among reported cases were similar to the male proportion in the population, differing by less that $1 \%$ (Table 1 ).

\section{Singapore}

Among the countries assessed, Singapore had the largest proportion of male dengue cases reported. The proportion of men among reported cases was significantly larger relative to the general population, ranging from $57 \%$ in the $5-14$ year age group to $61 \%$ in the $\geq 15$ year age group. The difference was significant in all age groups, except in infants where the numbers were small, with a consistent pattern over time from 1999 to 2005 (Table 1).

\section{Sri Lanka}

In Sri Lanka, there was a consistent and significantly larger proportion of males among reported dengue cases than expected in the $\geq 15$ year old age group from 1996 to 2005 (Table 1 ). This male excess was reported in every province (Table 2). Among 1-4 and 5-14 year olds, there were significantly fewer male cases than expected, although there was some annual variation. 
Dengue epidemiology in Sri Lanka underwent changes during 1996-2005. The proportion of reported cases from Western Province, which contains the country's largest city, Colombo, decreased from 84\% in 1999 to $37 \%$ in 2003 (data not shown). The age distribution of reported cases changed from children less than 15 years of age making up more than $60 \%$ of cases in 1996-1999 to less than $40 \%$ of cases in 2001-2005 (Table 1).

\section{Malaysia}

In Malaysia, only the total number of reported male and female dengue cases was available from 1997-2008 (Table 3 ). The majority of reported cases were persons over 15 years of age (between $76 \%$ and $82 \%$ for the years 1997-2008; data not shown). Although dengue data stratified for both age and sex were not available for Malaysia, the majority of reported cases were consistently male, significantly more than expected (Table 3). For each of the two years for which subnational data were available, there was a consistent pattern of excess in reported male cases from each state; for 2007, percent male ranged from $56 \%$ to $72 \%$ with an overall $59 \%$ and for 2008 , percent male ranged from $58 \%$ to $67 \%$ with an overall $62 \%$.

\section{Cambodia}

Dengue data for Cambodia were available for 2010. In Cambodia, 6116 of 12347 (49.6\%) cases reported in

Table 3. Total number of reported dengue cases and percent male among reported dengue cases, Malaysia, 1997-2008

\begin{tabular}{rrr}
\hline Year & Number of cases & \% male cases \\
\hline 1997 & 19429 & $54.4^{\star}$ \\
1998 & 27381 & $55.6^{\star}$ \\
1999 & 10146 & $59.6^{\star}$ \\
2000 & 7103 & $59.2^{\star}$ \\
2001 & 16368 & $56.2^{\star}$ \\
2002 & 32767 & $54.7^{\star}$ \\
2003 & 31545 & $56.1^{\star}$ \\
2004 & 33895 & $61.4^{\star}$ \\
2005 & 39686 & $58.1^{\star}$ \\
2006 & 38556 & $58.1^{\star}$ \\
2007 & 48846 & $59.3^{\star}$ \\
2008 & 49335 & $61.5^{\star}$ \\
\hline Percent male in Population & \\
\hline
\end{tabular}

*Denotes years and age groups for which the percent male among reported cases was significantly different from the percent male in the general population at the $\alpha=0.001$ level.

a Malaysia did not report male and female dengue cases by age group.

b Population data are for the year 2000 .
2010 (through 24 December 2010) were male. The proportion of reported male cases by age group were: $50.1 \%$ among those aged 4 years or less, $48.9 \%$ among those aged 5-14 years (significantly less than expected), and $60.1 \%$ among those aged 15 years or more (significantly more than expected) (Table 1 ).

\section{DISCUSSION}

This study, based on reported dengue cases from national surveillance systems, found a consistent and significant excess among males $\geq 15$ years of age. This pattern was consistent over a period of six to 10 years in three culturally and economically diverse countries and over geographically diverse subnational areas within two countries. Although age- and sex-stratified data were not available for Malaysia, most of reported cases were for persons over 15 years of age, and there was a consistent excess of male cases over a 12-year period. In a previous study of dengue incidence in Malaysia between 1973 and 1987 the majority of reported cases were also found to be male. ${ }^{9}$ The observed overall pattern of male excess among reported cases in older age groups agrees with previous studies in Singapore $e^{4,10,11}$ and has also been found in more recent dengue surveillance data from the Philippines for 2010 (the Philippines Department of Health, personal communication) and Singapore for 2009. ${ }^{12}$ Taken together, these findings suggest that there may be gender-related differences in dengue incidence, which might be due to exposure differences among older adolescents and adults. These results in Asia are in contrast to studies in South America, which have found either equal proportions of male and female dengue cases or a greater proportion of female cases..$^{5,7,13-15}$

The reasons for the excess of reported male dengue cases among older adolescents and adults in Asia observed in this study need further exploration. In Singapore, a careful analysis attributed greater reported male incidence of dengue from 1998 to 2000 to greater male exposures to dengue-carrying mosquitoes during daytime hours either at the workplace or while travelling to and from work. ${ }^{4}$ This theory is supported by the fact that aggressive public health measures in Singapore have been able to greatly reduce the mean number of mosquitoes in the home, ${ }^{10}$ and the fact that the labour force in Singapore has more males than females. ${ }^{4}$ However, this hypothesis has been questioned by a recent serological study of adults by Yew et al., ${ }^{11}$ which found no significant differences between males and 
females in recent dengue infection, despite the excess of male cases reported during the same year the serological study was conducted. Yew et al. suggested that malefemale differences in the use of health services and/ or male-female differences in disease severity might account for this discrepancy. For example, working adults in Singapore (who are more likely to be male) ${ }^{4,16}$ may be more likely to seek treatment and be reported to the Ministry of Health when ill because they require medical certification for absences.

Yew et al. did find males to be significantly more likely to have had a past dengue infection in a multivariate analysis which adjusted for ethnicity, age, and work status. ${ }^{11}$ The authors felt that the greater presence of males in the workforce could not explain this difference, however, since housewives, retirees and the unemployed were found to have proportionally larger seropositivity than those employed. Moreover, after adjusting for work status, male gender remained significantly associated with past infection status, which indicates that the higher seroprevalence could not be fully or explained by employment status alone. It was suggested that movement history should be studied as a possible contributing factor of dengue infection in Singapore.

For those $\leq 15$ years of age, sex differences in the reported number of cases were less striking. The differences found among those aged 1-4 years and 5-14 years were not consistent, and the magnitude of the difference was relatively small, except in Singapore. However, the excess number of male infants with dengue in the Philippines and Singapore deserves further study. Although there were relatively few reported cases among infants, the relatively high dengue case fatality during infancy makes infants an important risk group. Male excess in infants was also reported in more recent dengue surveillance data from the Philippines for 2010 (the Philippines Department of Health, personal communication) and for those aged 4 years or less from Singapore for 2009. ${ }^{12}$

Since the data assessed in this paper are based on national surveillance systems, they are subject to the limitations inherent in surveillance data such as underreporting, misreporting and reporting biases. Evidence from several studies suggests that only a minority of dengue infections are reported to surveillance systems, partly because a large proportion of dengue infections are either subclinical or asymptomatic. ${ }^{11}$
While differential reporting by sex is unlikely to fully explain the observed excess in adult males, any gender bias that exists in the use of health services could affect the number of reported male and female cases. For example, since adults appear to be more susceptible than children to developing symptoms after dengue virus infection, ${ }^{6,17,18}$ and if adult men are more likely to seek health care than adult women, there may be a larger number of reported adult male cases even if there is no difference in the underlying incidence rates. An important limitation was our inability to assess sex differences in reported dengue cases among adults more precisely. This was not ideal, particularly for comparing working adults and retirees. Since the epidemiology of dengue is rapidly changing, and has evolved into more of an adult disease rather than a paediatric disease in some countries, ${ }^{6,10}$ continued monitoring and assessment by appropriate age groups are important. Lastly, there are also likely to be variations over time and across countries in reporting practices and case definitions. While one of the aims of DengueNet was to harmonize case definitions, case definitions are not provided on DengueNet. Due to such variability, it is inappropriate to combine data from all countries. The fact that we found a consistent excess of males in the older age group across many years, and in both national and subnational data from several Asian countries, is an important strength of this study.

In conclusion, the current study found a consistent pattern of male predominance in the reported number of incident dengue cases among persons 15 years or older in several Asian countries. Since collapsing the data over all ages would have masked some of the observed differences, the findings indicate the importance of reporting sex and age stratified data for dengue surveillance. Assessment of how dengue differs for males and females by age is important because biological and gender-related factors can change over the human lifespan, and gender-related factors can differ across countries. Further research is required to identify the cause(s) of such sex-specific differences to target preventive measures to reduce the dengue burden in the region.

\section{Conflicts of interest}

None declared.

\section{Funding}

This study was undertaken as part of routine surveillance activities of the WHO Western Pacific Regional Office. 


\section{Acknowledgements}

The authors would like to thank the WHO Western Pacific Region Country Office staff for assisting with surveillance data collection and reporting. We would also like to thank Renu Dayal Drager and Richard Anker for their comments.

\section{References:}

1. Fact sheet $N^{\circ} 117$, Dengue and dengue hemorrhagic fever, March 2009. Geneva, World Health Organization (http://www.who. int/mediacentre/factsheets/fs117/en/, accessed 26 December 2010).

2. Dengue in the Western Pacific. Manila, World Health Organization Western Pacific Regional Office (http://www.wpro.who.int/health topics/dengue, accessed 20 December 2010).

3. Huy R et al. National dengue surveillance in Cambodia 19802008: epidemiological and virological trends and the impact of vector control. Bulletin of World Health Organization, 2010, 88(9):650-657. doi:10.2471/BLT.10.081729.

4. Eong $\mathrm{OE}$. Changing pattern of dengue transmission in Singapore. Dengue Bulletin, 2001, 25:40-4 (http://www.searo.who.int/ LinkFiles/Dengue_Bulletin_Volume_25_ch7.pdf, accessed 6 June 2011).

5. Kaplan JE et al. Epidemiologic investigations of dengue infection in Mexico, 1980. American Journal of Epidemiology, 1983, 117:335-343. pmid:6829561

6. Lin CC et al. Characteristic of dengue disease in Taiwan: 20022007. American Journal of Tropical Medicine and Hygiene, 2010, 82(4):731-739. doi:10.4269/ajtmh.2010.09-0549. pmcid:pmc2844571

7. Global Health Atlas. Geneva, World Health Organization (http:// apps.who.int/globalatlas/default.asp, accessed 26 December 2010).

8. World Population Prospects: The 2008 Revision, Highlights Working Paper No. ESA/P/WP.210. New York, United Nations Department of Economic and Social Affairs, 2009 (http:// www.un.org/esa/population/publications/wpp2008/wpp2008_ highlights.pdf, accessed 6 June 2011).

9. Shekhar KC, Huat OL. Epidemiology of dengue/dengue hemorrhagic fever in Malaysia-a retrospective epidemiological study 1973-1987. Part I: Dengue hemorrhagic fever (DHF). [Review]. Asia-Pacific Journal of Public Health, 1992-1993, 6:15-25. pmid:1308765

10. Ooi EE, Goh KT, Gubler DJ. Dengue prevention and 35 years of vector control in Singapore. Emerging Infectious Diseases, 2006, 12(6):887-893. pmid: 16707042

11. Yew YW et al. Seroepidemiology of dengue virus infection among adults in Singapore. Annals of the Academy of Medicine, Singapore, 2009, 38:667-675. pmid:19736569

12. Communicable Diseases Surveillance in Singapore 2009. Singapore Ministry of Health, 2010 (http://www.moh.gov.sg/ mohcorp/publicationsreports.aspx?id $=25254$, accessed 26 December 2010).

13. Günther J et al. Distribution of dengue cases in the state of Oaxaca, Mexico, during the period 2004-2006. Journal of Clinical Virology, 2009, 45:218-222. doi:10.1016/j.jcv.2009.05.007 pmid: 19487157

14. Amélia PA et al. Dengue epidemic in Belém, Pará, Brazil, 1996-1997. Emerging Infectious Diseases, 2000, 6(3). pmid: 10827121

15. García-Rivera EJ, Rigau-Pérez JG. Dengue severity in the elderly in Puerto Rico. Pan American Journal of Public Health, 2003, 13:362-368 (http://www.scielosp.org/scielo.php?script=sci arttext\&pid $=$ S1020-49892003000500004\&lng $=$ en\&nrm $=i s o \&$ tlng=en, accessed 6 June 2011).

16. Ooi EE et al. Dengue seroepidemiology in Singapore. Lancet. 2001, 3;357(9257):685-686. pmid:11247554

17. Endy TP et al. Epidemiology of inapparent and symptomatic acute dengue virus infection: a prospective study of primary school children in Kamphaeng Phet, Thailand. American Journal of Epidemiology, 2002, 156(1):40-51. doi:10.1093/aje/kwf005. pmid: 12076887

18. Burke DS et al. A prospective study of dengue infections in Bangkok. American Journal Tropical Medicine and Hygiene, 1988, 38(1):172-180. pmid:3341519 